\title{
Guidelines zum Mammakarzinom
}

\author{
Präambel
}

E. Eicher

1 Clinical practice guidelines for the care and treatment of breast cancer. A Canadian consensus document. CMAJ 1998;153 (3 Suppl.):S3-S8

2 Bundesamt für Statistik (Hrsg.). Statistisches Jahrbuch der Schweiz 2000. Zürich: NZZ; 1999.

3 Irwig L, Macashill P. Evidence relevant to guidelines for the investigation of breast symptoms. NHMRC National Breast Cancer Center 1997. ISBN 1876319046.

http://www.nbcc.org.au/pages/ info/resource/nbccpubs/

brcasymp/sec4.htm\#

\section{Einleitung}

Im weiten Bereich der Qualitätssicherung im Gesundheitswesen stellen Guidelines ein wichtiges und sinnvolles Segment dar, welches der Ärztin und dem Arzt in komplexen und sich rasch verändernden Fragestellungen in Diagnose, Therapie und medizinischer Technik eine wertvolle Hilfeleistung bietet. Guidelines sollen sich auf Evidenz basierten Publikationen und Expertenmeinungen abstützen und stellen damit den aktuellen Stand des Wissens dar. Sie gelten für den Regelfall; sie sind somit keine in jedem Einzelfall gültige Handlungsanweisungen und damit auch keine Untersuchungs- oder Behandlungsalgorithmen. Sie lassen der Ärztin und dem Arzt die Entscheidung offen, allenfalls von ihnen abzuweichen. Die individuelle Situation der Patientin, des Patienten - insbesondere bei Polymorbidität - ist zu berücksichtigen und zwingt unter Umständen dazu, von den Vorgaben einer Guideline abzuweichen. Ein solches Vorgehen kann und sollte begründet werden. Sofern eine Guideline gewisse qualitative Kriterien und Anforderungen erfüllt, ist sie ein geeignetes Mittel, den medizinischen Entscheidungsprozess zu erleichtern und damit auch die Qualität der ärztlichen Versorgung zu verbessern, auch wenn es äusserst schwierig ist, das Resultat der Anwendung einer Guideline zu evaluieren.

Die Erarbeitung einer Guideline erfordert Zeit, Geduld und viel Engagement einer Expertengruppe. Ist die Guideline nach den Prinzipien der Evidence-based Medicine und eines Expertenkonsensus erstellt, so müsste sie eigentlich weltweit immer identisch sein.

Gesundheitssysteme, Ausbildung der Ärztinnen und Ärzte, Behandlungstraditionen, ethische und religiöse sowie epidemiologische Unterschiede können jedoch eine Guideline beeinflussen, so dass sie nicht tel quel von einem anderen Land übernommen werden kann.

Im vorliegenden Fall haben die Arbeitsgruppen Aufbau und Kapiteleinteilung der Guideline der Canadian Medical Association [1] als Arbeitsgrundlage übernommen.

\section{Relevanz}

Das Mammakarzinom ist bei der Frau das am häufigsten vorkommende Karzinom. Der palpable Befund in der Mamma ist für die Praxis hoch relevant, genaue statistische Zahlen über dessen Vorkommen sind allerdings nicht verfügbar. Die Mortalität von Patientinnen mit Mammakarzinom betrug 1996 29,2 pro 100000 Personen [2].

\section{Ziel der diagnostischen Guideline}

Die Vereinheitlichung der Untersuchungsmethoden ist anzustreben. Damit kann die Verunsicherung der Patientinnen abgebaut werden. Noch nicht generell verbreitete Untersuchungsmethoden sind in dieser Guideline nicht berücksichtigt.

\section{Ziel der therapeutischen Guideline}

Der aktuelle Stand der verschiedenen Behandlungsmethoden soll aufgezeigt werden, über mögliche Kombinationen operativer, radioonkologischer und medikamentöser Therapien ist zu diskutieren.

\section{Methodik}

Die Verbindung der Schweizer Ärztinnen und Ärzte FMH hat in der Erkenntnis, dass aus den schon genannten Gründen Handlungsbedarf besteht, sämtliche involvierten Fachgesellschaften angeschrieben und gebeten, Experten in eine Arbeitsgruppe zu delegieren. Diese wurde durch die FMH koordiniert und geleitet. Die Experten für die diagnostische Guideline haben sich während 2 Jahren, diejenigen für den therapeutischen Teil während 3 Jahren regelmässig getroffen, die Literatur computerunterstützt gesucht mit MEDLINE ab 1966 und CANCER-LIT ab 1985 bis 2002; die relevante Literatur, insbesondere kürzlich publizierte Guidelines [1, 3], 
wurde durchgearbeitet und auf ihre Evidenz geprüft. Die Evidenzgrade der einzelnen Aussagen der Kurzfassung sind in den Begründungen der Empfehlungen mit $\varepsilon_{1}, \varepsilon_{2}, \varepsilon_{3}$ und $\varepsilon_{4}$ gekennzeichnet. Evidenzgrad $V\left(\varepsilon_{5}\right)$ entspricht dem Konsens der Arbeitsgruppe und wird nicht speziell vermerkt. Nach Fertigstellung der diagnostischen Guideline (6. Fassung) wurde sie an alle beteiligten Fachgesellschaften gesandt und durch je 10 Experten überprüft. Die Vernehmlassungsresultate wurden daraufhin erneut in der Expertengruppe diskutiert und in eine neue definitive Fassung gebracht. Eine klinische Testung wurde während 3 Monaten unter 60 Mitgliedern der verschiedenen involvierten Fachgesellschaften als letzter Schritt vor der definitiven Genehmigung durch die Experten ausgeführt.

\section{Grade der Evidenz \\ (Canadian Task Force on the Periodic Health Examination) [1]}

Grad I: Die Evidenz ist aufgrund randomisierter kontrollierter Studien (oder Metaanalysen) von genügendem Umfang derart, dass die Gefahr, dass sie falsch positive oder falsch negative Resultate beinhalten, gering ist.

Grad II: Die Evidenz basiert auf randomisierten kontrollierten Studien, welche jedoch zu klein sind, um ihnen Grad I zuzusprechen; sie können positive Trends, welche jedoch statistisch nicht signifikant sind, oder gar keine Trends zeigen. Sie sind mit einem hohen Risiko falsch negativer Resultate verbunden.

Grad III: Die Evidenz basiert auf nicht randomisierten Kontroll- oder Kohortenstudien, Fallserien, Fallkontrollstudien oder Querschnittsstudien.

Grad IV: Die Evidenz basiert auf der Meinung angesehener Experten oder Expertengremien, wie sie in publizierten Konsensuskonferenzen oder in Guidelines angegeben werden.

Grad V: Die Evidenz basiert auf der Meinung derjenigen Personen, welche diese Guidelines geschrieben oder aktualisiert haben, beruhend auf ihrer Erfahrung, ihrer Kenntnis der einschlägigen Literatur und der Diskussion mit ihren Fachkollegen.
Für die Erarbeitung der therapeutischen Guideline mussten folgende Untergruppen geschaffen werden:

- Mastektomie oder Tumorektomie;

- Axilladissektion;

- Carcinoma in situ;

- Radioonkologische Behandlung;

- Adjuvante Systemtherapie.

Das Vorgehen in diesen Untergruppen war dasselbe wie bei der diagnostischen Guideline mit der Ausnahme, dass auf die «klinische Testung» verzichtet wurde, teils aus Zeitmangel, teils aus der Erkenntnis, dass dieselben Experten der Fachgesellschaften mit dieser Aufgabe betraut würden, welche schon an der Vernehmlassung teilgenommen haben.

\section{Aktualisierung / Ausserkraftsetzung}

Die Guideline hat ab Veröffentlichung eine vorläufige Gültigkeit von 2 Jahren, muss aber bei Bedarf bereits vorher überarbeitet werden. Verantwortlich ist die Arbeitsgruppe unter Koordination der FMH.

\section{Dissemination und Implementierung}

Die Guideline steht auch im Internet zur Verfügung (www.emh.ch) und wird durch die Fachgesellschaften verbreitet. Die Guideline soll für Aus-, Weiter- und Fortbildungszwecke verwendet werden; weitere gedruckte Exemplare können bei der FMH bezogen werden.

\section{Finanzierung}

Bei der Erstellung der Guideline wurden keine Sponsorengelder eingesetzt, die Finanzierung der Expertengruppe, die Sekretariatsarbeiten, die Vervielfältigung der Literatur, erfolgte durch die FMH. Die jeweiligen Arbeitsfassungen wurden durch den Chefredaktor der Schweizerischen Ärztezeitung, Dr. Markus Trutmann, vorgenommen. 